\title{
Corporate Social Responsibility
}

\section{Liebe Leserinnen und Leser,}

seit einigen Jahren gewinnt ein Thema wieder neu an Bedeutung, das in der Tradition der Unternehmensführung in Deutschland eigentlich schon immer verankert war: die Verantwortung des Unternehmens gegenüber allen Stakeholdern, also neben den Aktionären auch Kunden, Mitarbeitern, Lieferanten, Umwelt und Gesellschaft. Im Zuge einer starken Fokussierung auf die Aktionäre war das Thema vielleicht hier und da etwas in den Hintergrund geraten; es wird angesichts mancher Exzesse im Management in den letzten Jahren aber wieder verstärkt diskutiert.

Mancher wird sich dabei nicht hinreichend sicher sein, ob Corporate Social Responsibility (CSR) nicht vielleicht doch nur ein Thema für die Öffentlichkeitsarbeit ist, das wenig mit dem Alltag in Controlling und Management zu tun hat. Soll das Thema aber nachhaltig ernst genommen werden, darf es in der Tat nicht auf Hochglanzbroschüren, Geschäftsberichte und immer neue Zertifizierungen reduziert werden, auch wenn dies noch vielfach der Fall ist. Vielmehr besteht Handlungsbedarf in zwei Richtungen: Zum einen gilt es, klare Spielregeln und Grenzen im Umgang mit den relevanten Stakeholdern zu definieren und nachzuhalten (etwa: „Keine Kinderarbeit bei unseren Zulieferern!“). Zum anderen müssen die wichtigsten Aspekte des verantwortungsvollen Umgangs mit den relevanten Stakeholdern gemessen, bewertet und immer dann in die laufende Unternehmenssteuerung integriert werden, wenn sie die Wertschöpfung in hinreichendem Umfang positiv oder negativ beeinflussen.

Bei beiden Aspekten ist das Controlling gefordert: Ein Controller, der sich als Business Partner versteht, wird sich dem Thema Corporate Social Responsibility somit auf Dauer kaum entziehen können. Controller müssen mit Blick auf die auferlegten Spielregeln entlang der ganzen Wertschöpfungskette dafür Sorge tragen, dass diese kontrollierbar sind, ohne dass das Unternehmen und die Zulieferer in einem weiteren Berg von formalen Kontrollen ersticken und ohne dass unternehmerische Initiative durch eine Absicherungskultur ersetzt wird. Zudem müssen sie transparent machen, wie viel die unter der Überschrift „Corporate Social Responsibility“ subsumierten Aktivitäten und Spielregeln kosten. Controller

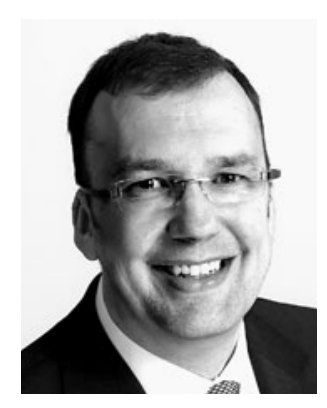

Utz Schäffer

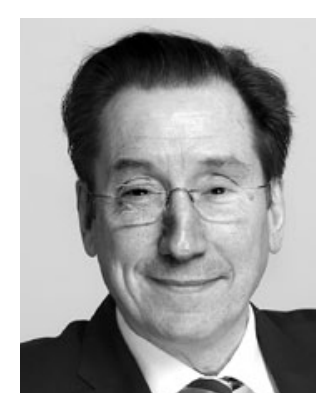

Jürgen Weber

müssen sich auch eine Meinung darüber bilden, welche Aspekte der Corporate Social Responsibility Treiber der Wertschöpfung des Unternehmens sind.

Häufig ist es dabei aber gar nicht so einfach zu sagen, wie teuer bestimmte Aktivitäten wirklich sind und ob sich eine bestimmte Spielregel oder Maßnahme nun rechnet oder nicht. Gerade im Bereich der Corporate Social Responsibility werden häufig weiche Faktoren berührt, die nur mit Mühe nachvollziehbar zu quantifizieren sind. Wie hoch ist die zusätzliche Zahlungsbereitschaft oder der zusätzliche Absatz wegen einer verbesserten Reputation, die sich etwa aus „grünem“ Handeln oder einem konsequenten Vermeiden von Kinderarbeit bei Zulieferunternehmen ergibt? Ab welchem Punkt verliert umgekehrt das Unternehmen die gesellschaftliche und ökonomische „licence to operate“, weil es das Thema Corpo- 
rate Social Responsibility nicht beachtet oder stark vernachlässigt hat?

Mit dem Schwerpunkt im vorliegenden Heft wollen wir Sie für das komplexe Thema sensibilisieren und einige Anregungen geben - erschöpfend behandeln können wir es in diesem Rahmen natürlich nicht. Die Empirie gibt uns übrigens Hoff- nung, dass eine solche Sensibilisierung gelingt: Unser WHUControllerpanel zeigt, dass in großen Unternehmen gerade die ersten Stellen für Nachhaltigkeitscontrolling eingerichtet werden!

Viel Spaß bei der Lektüre wünschen Ihnen
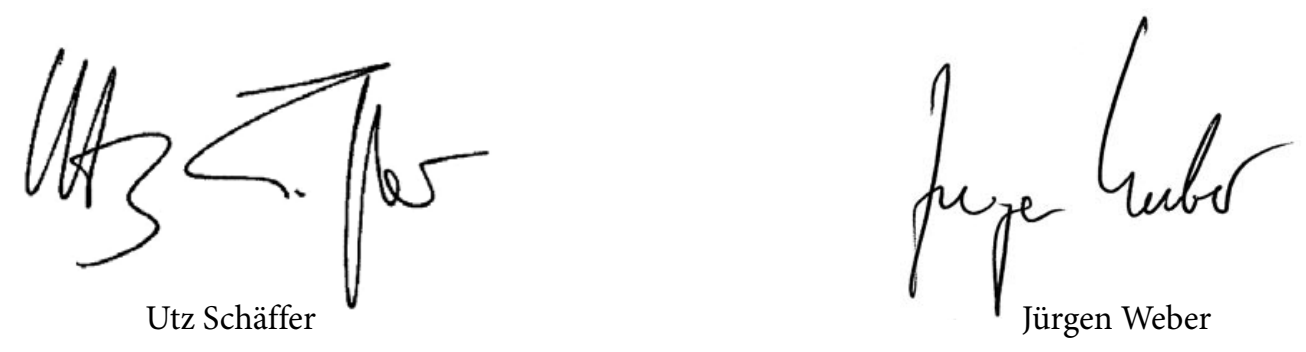


\section{EALTOR}

\section{WER NACHDENKT, LÖST PROBLEME. WER ABER VORAUSDENKT, ENTDECKT NEUE MÖGLICHKEITEN.}

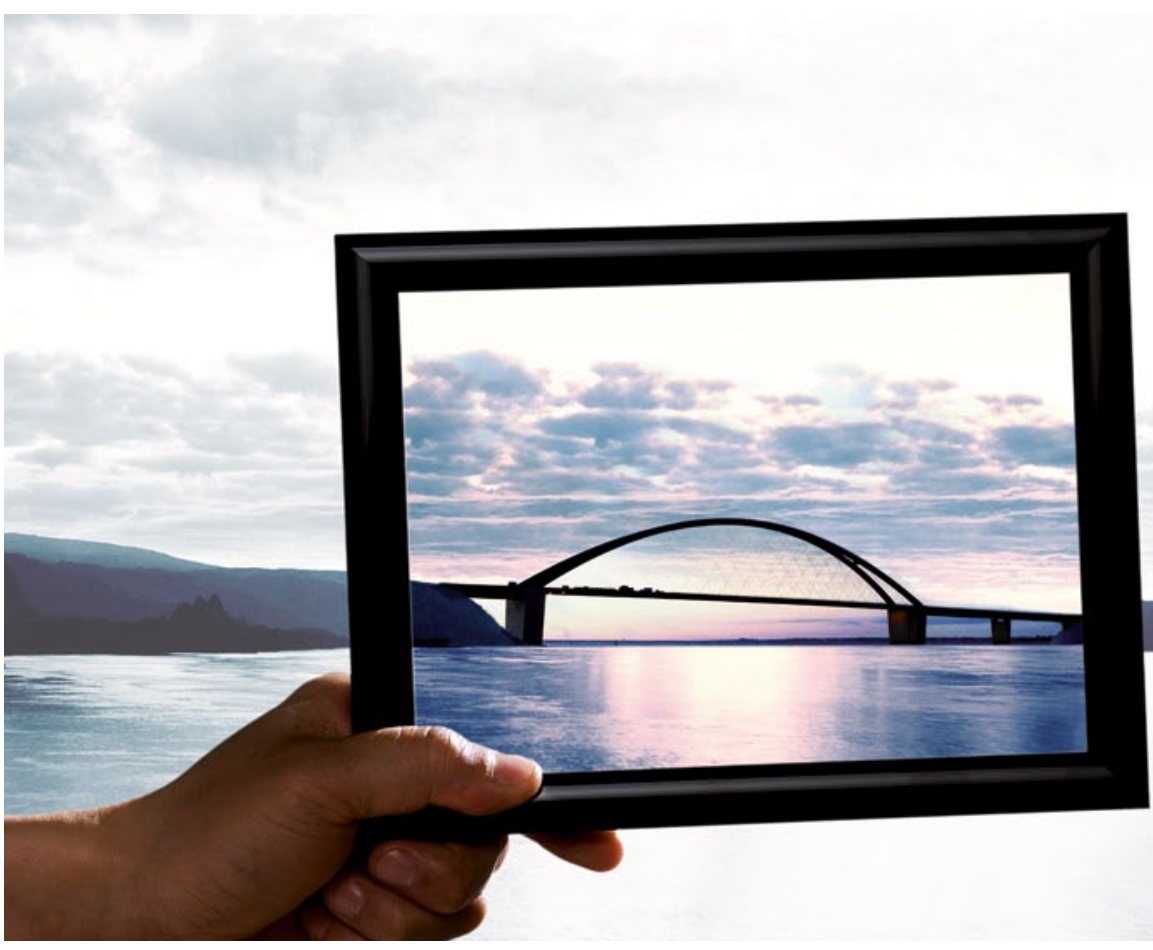

Wir erhalten Ihre Werte, Ihre Liquidität und sorgen dafür, dass Sie auch künftig wachsen und Ihre Ideen umsetzen können. Wie wir das tun? Indem wir dafür sorgen, dass Sie Ihr Geld schnell erhalten. Denn Außenstände sind gebundenes Kapital, das nicht für das Wachstum Ihres Unternehmens zur Verfügung steht.

Die ALTOR Gruppe verbindet die Unternehmen um die Heidelberger Inkasso zu einem der größten konzernunabhängigen Dienstleister für Forderungsmanagement in Deutschland.

Seit 1979 decken wir rund um das Thema Liquidität weite Phasen einer Kundenbeziehung ab von der Kündigungsprävention über die treuhänderische Bearbeitung notleidender Forderungen bis zu deren Ankauf. Exzellente Branchenexpertise, innovative Dienstleistungen und starke Partner unterstreichen unseren Service- und Beratungsanspruch.

ALTOR Gruppe, Im Breitspiel 13, 69126 Heidelberg, T 06221-987 654, www.altor-group.com 